\title{
Insights from the ENDO 2005 meeting
}

\author{
P Reed Larsen
}

I attended the very successful meeting of the American Endocrine Society in San Diego in June. There were approximately 6,500 delegates in attendance, about a third of these from outside the US. I was struck by the numbers of young people, particularly women, promising an energetic and diverse future for our subspecialty. The oral presentations, Meet-the-Professor clinical reviews, and the new scientific material were of high quality. The plenary lectures struck an excellent balance between basic research and clinical insights. A frequent problem at this meeting was deciding which of two interesting, but simultaneous, sessions to attend. Over the coming months, this journal will feature many of the novel findings presented at the meeting; here, space allows me to share only a few of the insights that this thyroidologist took away.

Ian Hay (Mayo Clinic, Rochester, USA) urged that we maintain a rational approach, based on the specific risk analysis at the time of diagnosis, to the treatment and follow-up of patients with papillary thyroid carcinoma; recent meta-analyses have not shown that radioactive iodine treatment improves outcome in these patients. Patient convenience, anxiety and cost issues dictate that the intensity of our surveillance is appropriate, given that only a relatively small fraction $(<15 \%)$ of these patients will have persistent disease or will succumb.

James Hennessey (Brown University, Providence, USA) reviewed the current controversies in the US over the definition of bioequivalence for levothyroxine (synthetic $\mathrm{T}_{4}$ ) preparations. The absence of comparative assays of therapeutic effectiveness including $\mathrm{TSH}$ as a biologic endpoint necessitates caution when theoretically equivalent generic levothyroxine preparations are substituted for a brand-name product, particularly in patients with thyroid cancer. Hopefully, continued efforts of the three major Endocrine Societies dealing with thyroid patients in the US will result in a meeting of minds with the FDA so that we

\section{A frequent \\ problem at \\ this meeting \\ was deciding \\ which of two \\ interesting, but \\ simultaneous, \\ sessions to \\ attend}

PR Larsen is

the Editor-in-

Chief of Nature

Clinical Practice

Endocrinology \&

Metabolism, and the

Chief of the Division

of Endocrinology,

Diabetes and

Hypertension at

Brigham and Women's

Hospital, Harvard

Medical School,

Boston, MA, USA.

\section{Competing interests}

The author declared he has no competing interests.

www.nature.com/clinicalpractice doi:10.1038/ncpendmet0050 can rest assured that our patients will always receive a biologically equivalent product.

Susan Mandel (University of Pennsylvania, Philadelphia, USA) emphasized the importance of careful monitoring of pregnant hyperthyroid women receiving antithyroid drugs to avoid overdosage with the potential consequence of fetal hypothyroidism. A minimal dose of antithyroid drug should be given, ignoring the suppressed TSH-the goal being to relieve clinical symptoms and signs but to allow normal fetal growth. The best biochemical parameter to follow is total serum $T_{4}$, which will usually remain slightly above the normal range for pregnancy. In a significant fraction of patients, the spontaneous decrease in thyroid-stimulating immunoglobulins during late gestation will obviate the need for antithyroid drugs in the last trimester.

The Allan-Herndon-Dudley syndrome, which is associated with mutations in a major thyroid-hormone transporter gene, SLC16A2 (MCT8), was reviewed by Theo Visser (Erasmus University, Rotterdam, The Netherlands) and Samuel Refetoff (University of Chicago, Chicago, USA) in a very exciting symposium. The $\mathrm{X}$-chromosome mutations found in all patients tested to date result in a mutant protein that does not transport $T_{3}$ or $\mathrm{T}_{4}$ into the cell. Apparently, impairment of thyroid-hormone uptake into critical structures in the human fetal brain during embryogenesis produces this striking syndrome, characterized by involuntary movements and impaired central-nervous-system development. What is unclear is why the phenotype of these patients is so different from that of congenital hypothyroidism. Could SLC16A2 also transport another molecule required for development of the central nervous system or is it the selectivity and timing of the deficit in $\mathrm{T}_{3}$ transport that is critical?

We look forward to future insights into these and many other new perspectives at the $88^{\text {th }}$ meeting of the American Endocrine Society here in Boston in June, 2006. 